“C 2016 IEEE. Personal use of this material is permitted. Permission from IEEE must be obtained for all other uses, in any current or future media, including reprinting/republishing this material for advertising or promotional purposes, creating new collective works, for resale or redistribution to servers or lists, or reuse of any copyrighted component of this work in other works." 


\title{
Electrically Small, Broadside Radiating Huygens Source Antenna Augmented with Internal Non-Foster Elements to Increase Its Bandwidth
}

\author{
Ming-Chun Tang, Senior Member, IEEE, Ting Shi, and Richard W. Ziolkowski, Fellow, IEEE
}

\begin{abstract}
A broadside radiating, linearly polarized, electrically small Huygens source antenna system that has a large impedance bandwidth is reported. The bandwidth performance is facilitated by embedding non-Foster components into the near-field resonant parasitic (NFRP) elements of this metamaterial-inspired antenna. High quality and stable radiation performance characteristics are achieved over the entire operational bandwidth. When the ideal non-Foster components are introduced, the simulated impedance bandwidth witnesses approximately a 17 -fold enhancement over the passive case. Within this $-10 \mathrm{~dB}$ bandwidth, its maximum realized gain, radiation efficiency, and front-to-back ratio (FTBR) are, respectively, $4.00 \mathrm{~dB}, 88 \%$, and $26.95 \mathrm{~dB}$. When the anticipated actual negative impedance convertor (NIC) circuits are incorporated, the impedance bandwidth still sustains more than a 10-fold enhancement. The peak realized gain, radiation efficiency, and FTBR values are, respectively, $3.74 \mathrm{~dB}, 80 \%$, and 28.01 dB, which are very comparable to the ideal values.
\end{abstract}

Index Terms-Directivity, electrically small antennas, front-to-back ratio, Huygens source antenna, impedance bandwidth, non-Foster elements.

\section{INTRODUCTION}

A $\mathrm{s}$ a consequence of their advantageous radiation performance characteristics for wireless applications, including high directivity with large front-to-back ratios (FTBRs) and wide beamwidths, electrically small Huygens source antennas have been investigated extensively in recent years [1-6]. However, because they are composed of pairs of electric and magnetic radiating elements that are electrically small in size and whose resonances must overlap with proper weighting, their impedance bandwidths are narrow and severely constrained by known passive bounds [7-9]. In recent years, non-Foster elements have been considered and utilized to

Manuscript received on Apr. 28, 2016; revised on Jul. 4, 2016...

This work was supported in part by the National Natural Science Foundation of China contract number 61471072, in part by graduate scientific research and innovation foundation of Chongqing, China contract numbers CYS16020, in part by the Fundamental Research Funds for the Central Universities contract number 106112015CDJZR165510, in part by China Postdoctoral Science Foundation contract number 2016M590860, and in part by Opening subject of State Key Laboratory of Millimeter Waves contract number K201732.

M. -C. Tang and T. Shi are with the College of Communication Engineering, Chongqing University, Chongqing, 400044, China and with the State Key Laboratory of Millimeter Waves, Nanjing, 210096, China (E-mail: tangmingchunuestc@126.com);

R. W. Ziolkowski is with The University of Arizona, Department of Electrical and Computer Engineering, Tucson, AZ 85721 USA and with the University of Technology Sydney, Global Big Data Technologies Centre, Ultimo NSW 2007, Australia (E-mail: ziolkowski@ece.arizona.edu). achieve much wider impedance bandwidths in electrically small antennas (ESAs), overcoming the traditional tradeoff drawbacks between impedance bandwidths, radiation efficiencies, and directivities [10-16].

In this letter, we numerically demonstrate that one can simultaneously augment the two pairs of near-field resonant parasitic (NFRP) electric and magnetic dipole elements of a Huygens source ESA [6] with corresponding pairs of properly designed internal non-Foster elements to achieve wideband operation while successfully maintaining its attractive radiation performance characteristics. All of the electromagnetic simulations were performed with the ANSYS/ANSOFT high frequency structure simulator (HFSS) and confirmed with the CST microwave studio (MWS) tools. The material parameters of all copper components: $\varepsilon_{\mathrm{r}}=1.0, \mu_{\mathrm{r}}=0.999991$ and bulk conductivity $\sigma=5.8 \times 10^{7} \mathrm{Siemens} / \mathrm{m}$, were properly taken into account. All of the circuit simulations were performed with Agilent's advanced design system (ADS).

\section{PASSIVE HuYGENS SOURCE ESA}

The design principles of a realized low-profile, broadside radiating, high-FTBR Huygens source ESA were reported in [6]. An evolutionary pair of Egyptian axe dipoles (EADs) and a pair of capacitively loaded loops (CLLs) serve as the electric and magnetic NFRP elements coupled to a driven printed dipole antenna. The updated version of this experimentally confirmed configuration, which is reported here, is shown in Fig.1; and its optimized parameters are given in Table I. All the substrates used were Rogers Duroid ${ }^{\mathrm{TM}} 5880$ with 0.5 oz (17 $\mu \mathrm{m})$ copper cladding. The substrate thickness with the CLLs on it was $0.508 \mathrm{~mm}$; the remaining substrates were $0.787 \mathrm{~mm}$ thick. As shown in Figs. 1(a) and 1(b), a $50 \Omega$ coaxial cable was included in the model to enhance the simulation accuracy. It is noted that the EAD (electric) pairs and CLL (magnetic) pairs are orthogonally oriented and have vertical placements that facilitate the overlapping of their phase centers.

Two pairs of capacitors and resistors in series were embedded into the rectangular slots at the center positions of both the CLL and the EAD NFRP elements as shown in Fig. 1(a). As illustrated in Fig. 1(b), one capacitor: $C_{m}=1.6 \mathrm{pF}$, and one resistor: $R=10^{-5} \Omega$, were placed in series in each centered gap of the CLLs. Similarly, one capacitor: $\mathrm{C}_{\mathrm{e}}=1.5 \mathrm{pF}$, and one resistor: $\mathrm{R}=10^{-5} \Omega$, were placed in series in each centered gap of the EADs as depicted in Fig. 1(c). Note that the resistance value, which is very close to but not equal to zero, was 
introduced simply to suppress any potential runtime software errors associated with the HFSS simulations.
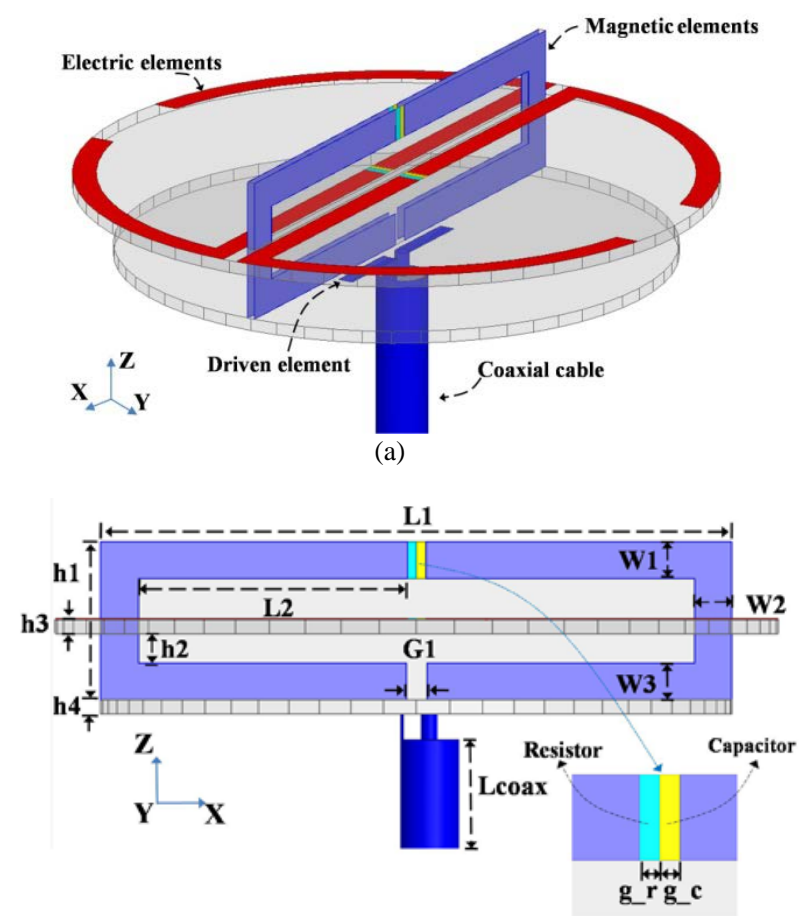

(b)

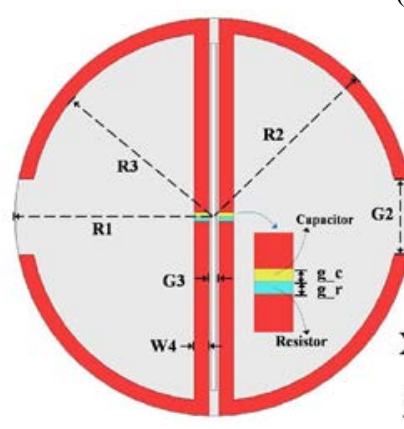

(c)

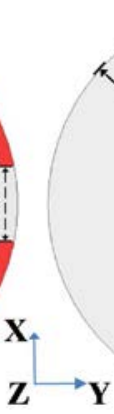

$\mathbf{Z} \longrightarrow \mathbf{Y}$

(d)
Fig. 1. The Huygens source ESA; (a) 3-D isometric view, (b) side view of the magnetic NFRP elements, (c) top view of the electric NFRP elements, and (d) bottom view of the driven, printed dipole element.

TABLE I. Optimized PAsSive Huygens Source ESA PARAMETERS (IN MM)

\begin{tabular}{|c|c|c|c|}
\hline$L 1=35.8$ & $L 2=15.3$ & $L 3=5.5$ & $L$ coax $=5$ \\
\hline$W 1=2.1$ & $W 2=2.1$ & $W 3=2.1$ & $W 4=1.5$ \\
\hline$W 5=1$ & $h 1=9.5$ & $h 2=1.72$ & $h 3=0.787$ \\
\hline$h 4=0.787$ & $R 1=20.5$ & $R 2=20.5$ & $R 3=18.98$ \\
\hline$R 4=16.7$ & $G 1=1.15$ & $G 2=7.9$ & $G 3=1$ \\
\hline$g_{-} r=0.5$ & $g_{-} c=0.5$ & \multicolumn{2}{|c|}{ Null } \\
\hline
\end{tabular}

Fig. 2 gives the simulated performance characteristics of this passive Huygens source ESA. The resonant frequency is $f_{\text {res }}=$ $1.580 \mathrm{GHz}$ with $\left|\mathrm{S}_{11}\right|_{\min }=-42.62 \mathrm{~dB}$, and its $-10 \mathrm{~dB}$ fractional bandwidth (FBW) (i.e., set of frequencies with $\left|\mathrm{S}_{11}\right| \leq-10 \mathrm{~dB}$ ) is very narrow: $0.60 \%$. Accordingly, its total height and electrical size $k a$ (where $a$ is the radius of the smallest sphere that completely encloses the antenna system at its operational wavelength $\lambda_{0}$, and where $k=2 \pi / \lambda_{0}=2 \pi f_{\text {res }} / c$ is the free space wave number) are, respectively, $0.05 \lambda_{0}$ and 0.68 . Compared with the Huygens source ESA design with a single CLL element reported in [6], the pair of CLLs in parallel placement in the present design enabled enhanced broadside radiation performance, i.e., the peak realized gain, radiation efficiency, and FTBR are increased, respectively, from 3.30 to $4.03 \mathrm{~dB}$, from 71.6 to $82.74 \%$, and from 18.76 to $20.21 \mathrm{~dB}$. Note that the simulated maximum cross-polarization levels in both planes were lower than $-42.25 \mathrm{~dB}$ and, consequently, are not plotted in Fig. 2(b). These HFSS results were confirmed with corresponding CST simulations.

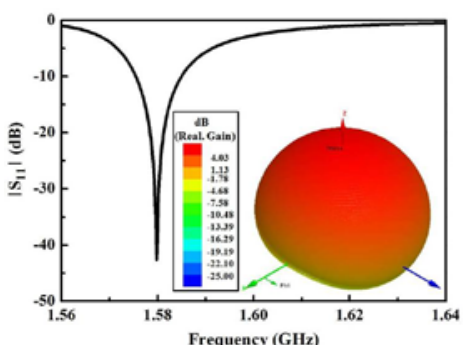

(a)

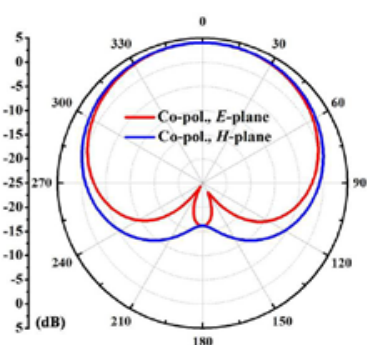

(b)
Fig. 2. The Huygens source ESA performance characteristics. (a) $\left|S_{11}\right|$ values as a function of the frequency and the 3-D realized gain pattern at $\mathrm{f}_{\mathrm{res}}$, and (b) the 2-D realized gain patterns in the $\mathrm{E}$ - and $\mathrm{H}$ - planes at $\mathrm{f}_{\mathrm{res}}=1.580 \mathrm{GHz}$.

\section{The IdEAL Non-Foster Huygens SOURCE ESA}

Following the non-Foster augmentation design strategies reported in [11-13], the frequency-agile performance of the passive Huygens source ESA was first determined by varying the $\mathrm{C}_{\mathrm{m}}$ and $\mathrm{C}_{\mathrm{e}}$ values. In particular, by sweeping $\mathrm{C}_{\mathrm{m}}\left(\mathrm{C}_{\mathrm{e}}\right)$ from 1.2 (1.39) to $2.4 \mathrm{pF}(1.85 \mathrm{pF})$ in $0.1 \mathrm{pF}$ steps, one obtains the simulated values summarized in Fig. 3 . It is clear that the FTBR values are higher than $7.8 \mathrm{~dB}$ for each of these frequency-agile states and $\left|S_{11}\right|_{\min }$ is much lower than -20 dB.

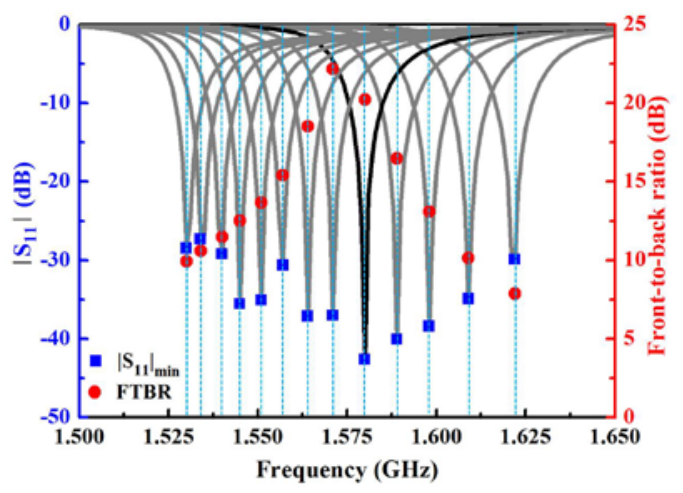

Fig. 3. The frequency-agile characteristics of the Huygens source ESA.

The combinations of these $\mathrm{C}_{\mathrm{m}}$ and $\mathrm{C}_{\mathrm{e}}$ values that produce the peak FTBR values are represented by the frequency-agile (blue square) marks shown in Fig. 4. In order to capture these requisite responses with the NFRP elements augmented with ideal non-Foster circuits, the equation $C\left(f_{\text {res }}\right)=a_{0}+a_{1} / f_{\text {res }}+$ $a_{2} / f_{\text {res }}{ }^{2}$ was used as the curve-fit to these discrete $C_{m}$ and $C_{e}$ values [11]. The calculations of the coefficients in each case were carried out using the curve-fitting tools in Matlab R2010b. We determined $C_{m}\left(f_{\text {res }}\right)=128.12-430 / f_{\text {res }}+363.55 / f_{\text {res }}{ }^{2}$ for the CLL elements, and $C_{e}\left(f_{\text {res }}\right)=134.0-430 / f_{\text {res }}+348.52 / f_{\text {res }}{ }^{2}$ for the EAD elements. Notice that in contrast to this curve-fit equation, $L\left(f_{\text {res }}\right)$ or $C\left(f_{\text {res }}\right)$ was expressed by $a_{0}+a_{2} / f_{\text {res }}{ }^{2}$ for a single NFRP ESA in [11]. The extra term: $a_{1} / f_{\text {res }}$, in both the $C_{m}$ 
and $\mathrm{C}_{\mathrm{e}}$ curve-fits, is introduced to account for the unavoidable capacitive coupling between the electric and the magnetic NFRP elements. The results defined by the curve-fit equations are represented by the solid red curves in Fig. 4 for easy comparison. These ideal values are the ones used to design the realistic NIC circuits.

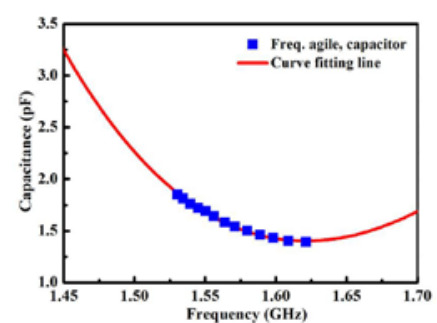

(a)

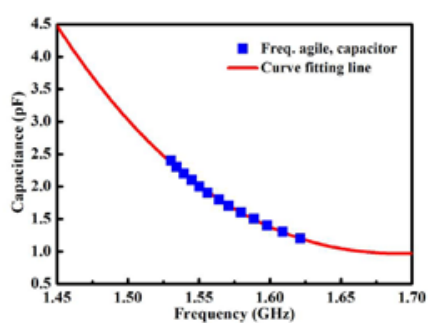

(b)
Fig. 4. Comparison between the discrete capacitance values and their curve-fit lines as functions of the resonance frequency $f_{\text {res }}$. (a) $C_{e}$ and (b) $C_{m}$ values.

By importing these $C_{m}$ and $C_{e}$ equation curves into the HFSS and CST models, the radiation performance characteristics of the Huygens source ESA augmented with the ideal non-Foster elements were simulated. The results are summarized in Fig. 5. The presence of the ideal non-Foster elements enhances the -10dB FBW from $0.6 \%$ up to $10.04 \%$, a 16.73 -fold improvement. Within this operational bandwidth, the peak realized gain values (strictly along the $+\mathrm{z}$-axis) fluctuate in the range $2.41-4.0 \mathrm{~dB}$, less than a $2 \mathrm{~dB}$ variation. The associated radiation efficiency and FTBR values are, respectively, in the ranges: $79-88 \%$ and $4.61-26.95 \mathrm{~dB}$, confirming that the ideal non-Foster augmentation of the broadside radiating ESA has significantly improved its bandwidth while maintaining (and even enhancing) its radiation performance characteristics.

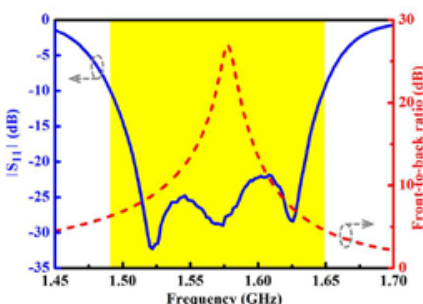

(a)

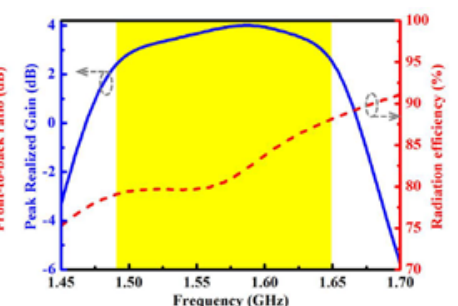

(b)
Fig. 5. Performance characteristics of the ideal non-Foster Huygens source ESA. (a) $\left|S_{11}\right|$ and FTBR values versus frequency, and (b) peak realized gain and radiation efficiency values versus frequency. (The $-10 \mathrm{~dB}$ impedance bandwidth range is highlighted in yellow.)

\section{The ReAlistic Non-Foster HuYgens SourCE ESA}

A negative impedance converter (NIC) circuit was designed to reproduce as close as possible the ideal curve-fits to both the $\mathrm{C}_{\mathrm{e}}$ and $C_{m}$ values. It was based on the floating NIC design introduced in [12]. The ADS model of the resulting NIC circuit is shown in Fig. 6. The BJT transistors, pb_hp_AT41533, available in the ADS component library, were employed. The lumped element $L, C$, and $R$ values were optimized numerically; the final parameter values are listed in Tables II(a) and II(b). The resulting $C_{e}\left(f_{\text {res }}\right)$ and $C_{m}\left(f_{\text {res }}\right)$ values are shown, respectively, in Figs. 7(a) and 7(b). While they were very close to their ideal capacitance values, the appearance of non-zero resistance values, $R_{e}$ and $R_{m}$, was inevitable, i.e., there are losses associated with all of the components. Since any large resistance values could lower the radiation efficiency significantly, the minimization of $R_{e}$ and $R_{m}$ arising from the embedded NIC circuit was necessary and a fundamental aspect of the optimization process. This proved difficult because tradeoffs exist between the resistances and the accuracy of the curve-fit capacitance values over the frequency range of interest. As is shown in Figs. 7(a) and (b), both the $R_{e}$ and $R_{m}$ values were optimized to be very small and non-negative to avoid any instability issues (as in [12], stability was tested with time domain simulations). Note that the values L1 and L2, and R3 and R4 are not identical. Their slight differences provided additional freedom for the NIC circuit to more accurately reproduce the ideal curve-fit capacity values.

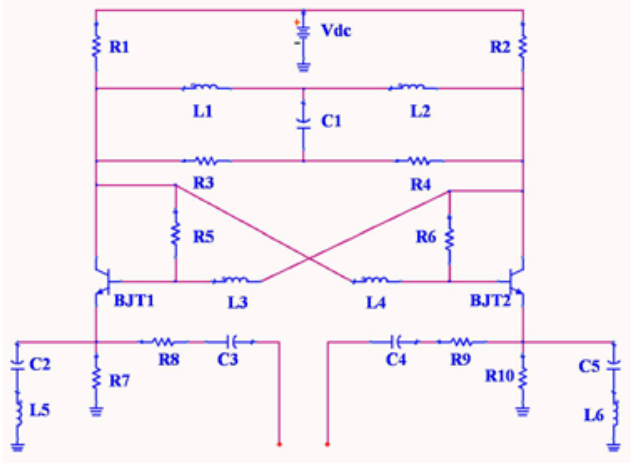

Fig. 6. Circuit model of the negative capacitor two-port floating NIC element.

TABLE II. OPTIMIZED COMPONENT VALUES

(a) NIC circuits embedded into the EADs

\begin{tabular}{|c|c|c|c|}
\hline$V d c=33.1 \mathrm{~V}$ & $R 1=5.78 \mathrm{k} \Omega$ & $R 2=145.8 \mathrm{k} \Omega$ & $R 3=9 \Omega$ \\
\hline$R 4=4.383 \mathrm{k} \Omega$ & $R 5=904 \Omega$ & $R 6=1.144 \mathrm{k} \Omega$ & $R 7=3.773 \mathrm{k} \Omega$ \\
\hline$R 8=2.05 \Omega$ & $R 9=2.05 \Omega$ & $R 10=0.99 \Omega$ & $C 1=0.141 \mathrm{pF}$ \\
\hline$C 2=0.05 \mathrm{pF}$ & $C 3=8.12 \mathrm{pF}$ & $C 4=8.12 \mathrm{pF}$ & $C 5=2.31 \mathrm{pF}$ \\
\hline$L 1=92.2 \mathrm{nH}$ & $L 2=98.5 \mathrm{nH}$ & $L 3=41.1 \mathrm{nH}$ & $L 4=17.7 \mathrm{nH}$ \\
\hline$L 5=1043.5 \mathrm{nH}$ & $L 6=3.92 \mathrm{nH}$ & \multicolumn{2}{|c|}{ Null } \\
\hline
\end{tabular}

(b) NIC circuits embedded into the CLLs

\begin{tabular}{|c|c|c|c|}
\hline$V d c=28.1 \mathrm{~V}$ & $R 1=5.86 \mathrm{k} \Omega$ & $R 2=56.07 \mathrm{k} \Omega$ & $R 3=6.95 \mathrm{k} \Omega$ \\
\hline$R 4=5.218 \mathrm{k} \Omega$ & $R 5=561 \Omega$ & $R 6=900 \Omega$ & $R 7=4.48 \mathrm{k} \Omega$ \\
\hline$R 8=3.645 \Omega$ & $R 9=3.645 \Omega$ & $R 10=5.5 \Omega$ & $C 1=30.58 \mathrm{pF}$ \\
\hline$C 2=0.38 \mathrm{pF}$ & $C 3=4.92 \mathrm{pF}$ & $C 4=4.92 \mathrm{pF}$ & $C 5=11.9 \mathrm{pF}$ \\
\hline$L 1=4.63 \mathrm{nH}$ & $L 2=914 \mathrm{nH}$ & $L 3=25 \mathrm{nH}$ & $L 4=14.7 \mathrm{nH}$ \\
\hline$L 5=58.42 \mathrm{nH}$ & $L 6=0.04 \mathrm{nH}$ & \multicolumn{2}{|c|}{ Null } \\
\hline
\end{tabular}

Incorporating the $C_{e}$ and $C_{m}$ NIC elements into the antenna system, the simulated performance characteristics shown in Figs. 8 and 9 were obtained. Fig. 8 gives the $\left|S_{11}\right|$, FTBR, realized gain, and radiation efficiency values as functions of the excitation frequency. Taking the center frequency to be 1.574 $\mathrm{GHz}$ (GPS L1), the -10dB impedance bandwidth was $94 \mathrm{MHz}$, from 1.536-1.63 GHz (5.97 \% FBW). Thus, a 10.03-fold increase over the impedance bandwidth of the passive version was realized. The realized gain, FTBR, and radiation efficiency values fluctuated, respectively, in the ranges: $0.04-3.74 \mathrm{~dB}$, 4.61-26.945 dB, and 42.5-80\%. These are comparable to the ideal Huygens source ESA valuations.

It should be noted that despite their low values, the resistances of the actual NIC circuit do have an impact on the radiation characteristics of the entire antenna system and 
cannot be neglected. This is a consequence of the intense resonance behavior of the system. Moreover, when compared to the ideal non-Foster results, the actual accuracy of the curve-fits obtained with the NIC circuit is what limits the obtainable impedance bandwidth.

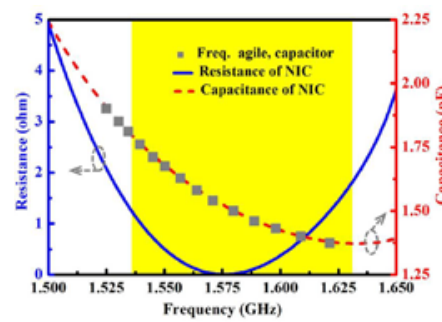

(a)

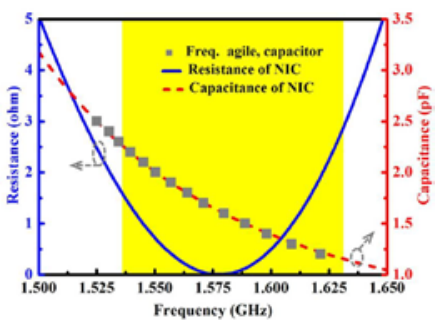

(b)
Fig. 7. Comparison between the capacitance and resistance values produced by the NIC circuits and their requisite frequency agile values. (a) EAD, and (b) CLL elements.

Figs. 9 (a)-(c) show, respectively, the 3-D realized gain patterns at the lower edge, middle, and higher edge of the frequencies in the $-10 \mathrm{~dB}$ impedance bandwidth of the capacitive NIC-loaded Huygens source ESA. They illustrate its stable, broadside radiating (maximum strictly along the +z-axis), and high FTBR behaviors across that frequency range. Finally, a comprehensive comparison between the passive, ideal non-Foster, and the actual NIC-loaded versions of the Huygens source antennas is provided in Table III.

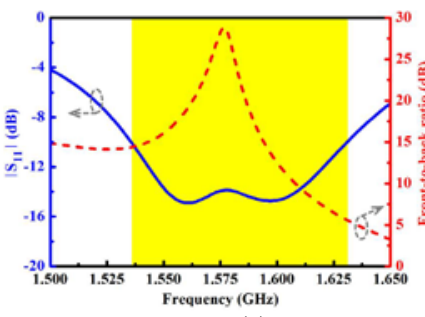

(a) (b)

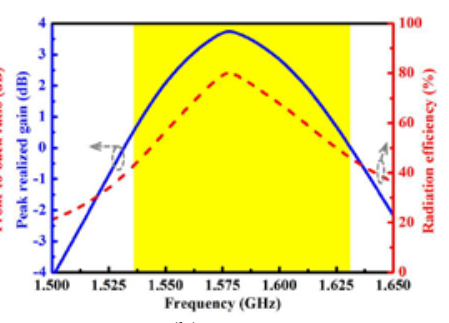

Fig. 8. Radiation performance characteristics of the NIC-loaded Huygens source ESA. (a) $\left|\mathrm{S}_{11}\right|$ and FTBR values, and (b) peak realized gain and radiation efficiency values versus the excitation frequency. (The $-10 \mathrm{~dB}$ impedance bandwidth region is highlighted in yellow.)

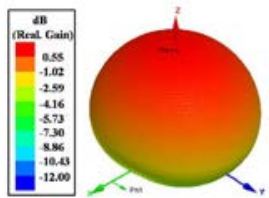

(a)
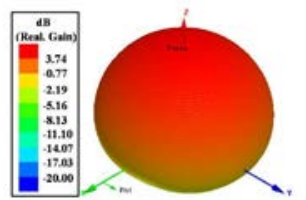

(b)
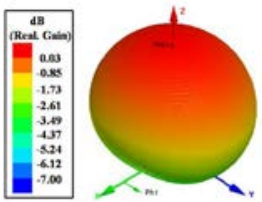

(c)
Fig. 9. The 3 D realized gain patterns of the NIC-loaded Huygens source ESA at the frequencies: (a) 1.536, (b) 1.58, and (c) $1.631 \mathrm{GHz}$.

\section{CONCLUSIONS}

A non-Foster, low-profile, broadside radiating, high FTBR, efficient, Huygens source ESA was demonstrated. By embedding ideal non-Foster components into the NFRP elements, a 16.73-fold enhancement of the impedance bandwidth was achieved. When two pairs of realizable capacitive NIC elements were incorporated into the Huygens source ESA system, a 10-fold bandwidth enhancement was obtained. In both cases, the non-Foster augmented ESAs exhibit excellent, stable broadside radiation performance characteristics throughout their entire operational bandwidths. We anticipate that our future fabrication and testing campaign will be able to acquire quality components (e.g., RF inductors are commercially available with sufficiently large inductance values) to realize the reported designs.

TABLE III. COMPARISON OF PERFORMANCE CHARACTERISTICS

\begin{tabular}{|c|c|c|c|}
\hline Category & $\begin{array}{c}\text { Passive } \\
\text { version }\end{array}$ & $\begin{array}{c}\text { Ideal } \\
\text { non-Foster } \\
\text { version }\end{array}$ & $\begin{array}{c}\text { Actual NIC } \\
\text { loaded } \\
\text { version }\end{array}$ \\
\hline$f_{\text {res }}(\mathrm{GHz})$ & 1.580 & & \\
\hline$\left|\mathrm{S}_{11}\right|_{\min }(\mathrm{dB})$ & -42.62 & & \\
\hline FWB (\%) & 0.60 & 10.04 & 6.02 \\
\hline Enhanced times & & 16.73 & 10.03 \\
\hline FTBR (dB) & 20.21 & $4.61-26.95$ & $5.63-28.01$ \\
\hline Radiation efficiency (\%) & 82.74 & $79-88$ & $42.5-80.0$ \\
\hline Peak Real. Gain (dB) & 4.03 & $2.41-4.00$ & $0.04-3.74$ \\
\hline
\end{tabular}

\section{REFERENCES}

[1] S. R. Best, "Progress in the design and realization of an electrically small Huygens source," in Proc. 2010 International Workshop on Antenna Technology (iWAT), Lisbon, Portugal, Mar. 1-3, 2010.

[2] P. Jin and R. W. Ziolkowski, "Metamaterial-inspired, electrically small Huygens sources,” IEEE Antennas Wirel. Propag. Lett., vol. 9, pp. 501-505, 2010.

[3] P. Alitalo, A. O. Karilainen, T. Niemi; C. R. Simovski, S. A. Tretyakov, "Design and realisation of an electrically small Huygens source for circular polarization," IET Microw. Antennas Propag., vol. 5, no. 7, pp. 783-789, 2011.

[4] T. Niemi, P. Alitalo, A. O. Karilainen, and S. A. Tretyakov, "Electrically small Huygens source antenna for linear polarization," IET Microw. Antennas Propag., vol. 6, no. 7, pp. 735-739, 2012.

[5] R. W. Ziolkowski, "Low profile, broadside radiating, electrically small Huygens source antennas," IEEE Access, vol. 3, pp. 2644-2651, Dec. 2015.

[6] M.-C. Tang, H. Wang, and R. W. Ziolkowski, "Design and testing of simple, electrically small, low-profile, Huygens source antennas with broadside radiation performance," IEEE Trans. Antennas Propag., submitted for publication, Jan. 2016.

[7] L. J. Chu, "Physical limitations of omni-directional antennas," J. Appl. Phys., pp. 1163-1175, Dec. 1948.

[8] A. D. Yaghjian and S. R. Best, "Impedance, bandwidth, and Q of antennas," IEEE Trans. Antennas Propag., pp. 1298-1324, Apr. 2005.

[9] M. Gustafsson, C. Sohl, and G. Kristensson, "Illustrations of new physical bounds on linearly polarized antennas," IEEE Trans. Antennas Propag., vol. 57, no. 5, pp. 1319-1327, May 2009.

[10] S. E. Sussman-Fort, and R. M. Rudish, "Non-Foster impedance matching of electrically-small antennas," IEEE Trans. Antennas Propag., vol. 57, no.8, pp. 2230-2241, Aug. 2009.

[11] P. Jin and R. W. Ziolkowski, "Broadband, efficient, electrically small metamaterial-inspired antennas facilitated by active near-field resonant parasitic elements,” IEEE Trans. Antennas Propag., vol. 58, no. 2, pp. 318-327, Feb. 2010.

[12] N. Zhu and R. W. Ziolkowski, "Broad bandwidth, electrically small, non-Foster element-augmented antenna designs, analyses, and measurements," IEICE Transactions on Communications, vol. E96-B, no.10, pp. 2399-2409, Oct. 2013.

[13] R. W. Ziolkowski, M.-C. Tang and N. Zhu, "An efficient, broad bandwidth, high directivity, electrically small antenna," Microw. Opt. Technol. Lett., vol. 55, no. 6, pp. 1430-1434, June 2013.

[14] C. R. White, J. S. Colburn, and R. G. Nagele, "A non-Foster VHF monopole antenna," IEEE Antennas Wireless Propag. Lett., vol. 11, pp. 584-587, 2012.

[15] M. Barbuto, A. Monti, F. Bilotti, and A. Toscano, "Design of a non-Foster actively loaded SRR and application in metamaterial-inspired components," IEEE Trans. Antennas Propag., vol. 61, no. 3, 1219-1227, Mar. 2013.

[16] H. Mirzaei and G. V. Eleftheriades, "A resonant printed monopole antenna with an embedded non-Foster matching network," IEEE Trans. Antennas Propag., vol. 61, no. 11, pp. 5363-5371, Nov. 2013. 